The authors of Zhang et al. 34:1446-1449 (2021) retracted this article because the initially reported genome assembly of NAFU1 was contaminated. This article was retracted on 28 November 2022, and the correction of the NAFU1 genome assembly at NCBI will be made in the future.

\title{
Genome Sequence Resource for Erysiphe necator NAFU1, a Grapevine Powdery Mildew Isolate Identified in Shaanxi Province of China
}

\author{
Xingyuan Zhang, ${ }^{1,2}$ Bo Mu, ${ }^{1,2}$ Kaicheng Cui, ${ }^{1,2}$ Min Liu, ${ }^{1,2}$ Guihua Ke, ${ }^{1,2}$ Yongtao Han, ${ }^{1,2}$ \\ Ying $\mathrm{Wu}^{3}{ }^{3}$ Shunyuan $\mathrm{Xiao}^{3}$ and Ying-Qiang Wen ${ }^{1,2, \dagger}$ \\ ${ }^{1}$ State Key Laboratory of Crop Stress Biology for Arid Areas, College of Horticulture, Northwest \\ A\&F University, Yangling, 712100, Shaanxi, China \\ ${ }^{2}$ Key Laboratory of Horticultural Plant Biology and Germplasm Innovation in Northwest China, \\ Ministry of Agriculture and Rural Affairs, Yangling, 712100, Shaanxi, China \\ ${ }^{3}$ Institute for Bioscience and Biotechnology Research \& Department of Plant Sciences and \\ Landscape Architecture, University of Maryland College Park, Rockville, MD 20850 U.S.A.
}

\begin{abstract}
Erysiphe necator is an economically important biotrophic fungal pathogen responsible for powdery mildew disease on grapevine. Currently, genome sequences are available for only a few $E$. necator isolates from the United States. Based on the combination of Nanopore and Illumina sequencing technologies, we present here the complete genome assembly for an isolate of $E$. necator, NAFU1, identified in China. We acquired a total of $15.93 \mathrm{~Gb}$ of raw reads. These reads were processed into a $61.12-\mathrm{Mb}$ genome assembly containing 73 contigs with an $\mathrm{N}_{50}$ of $2.06 \mathrm{Mb}$ and a maximum length of $6.05 \mathrm{Mb}$. Combining the results of three gene-prediction modules (i.e., an evidence-based gene modeler [EVidenceModeler], an ab initio gene modeler, and a homology-based gene modeler), we predicted 7,235 protein-coding genes in the assembled genome of $E$. necator NAFU1. This information will facilitate studies of genome evolution and pathogenicity mechanisms of $E$. necator and other powdery mildew species through comparative genome sequence analysis and other molecular genetic tools.
\end{abstract}

\section{Genome Announcement}

Powdery mildew caused by ascomycete fungi in the order Erysiphales is an important and widespread disease of numerous plant species, including wheat, barley, bean, rose, rubber, tomato, strawberry, and grape (Braun and Cook 2012; Wu et al. 2018). The Eurasian grape, Vitis vinifera L., is the most widely cultivated and economically important fruit crop in the world. However, the Eurasian grape is susceptible to many oomycete and fungal diseases, including downy mildew caused by Plasmopora viticola, gray mold caused by Botrytis cinerea, and powdery mildew caused by Erysiphe necator (Gadoury et al. 2012; Gessler et al. 2011). Among these three types of pathogens, E. necator does not require specific humidity and temperature conditions for infection (Dry et al. 2010); therefore, powdery mildew is the most frequent disease of grapevines in many areas. E. necator can infect all green tissues of a grapevine. As in other plants, grapevine leaves infected by powdery mildew often

${ }^{\dagger}$ Corresponding author: Y.-Q. Wen; wenyq@nwsuaf.edu.cn

X. Zhang and B. Mu contributed equally to this study.

The author(s) declare no conflict of interest.

Accepted for publication 24 August 2021. Retracted 28 November 2022.

\section{Funding}

This work was supported by the National Natural Science Foundation of China (grant numbers 31772264 and 31972986) and the National Key Research and Development Program of China (2018YFD1000300) to

Y.-Q. Wen and National Science Foundation support (IOS-1901566) to S. Xiao.

\section{Keywords}

Erysiphe necator, genome, genomics, grapevine, powdery mildew 
show reduced photosynthesis and suffer from premature senescence and abscission (Han et al. 2016). Even a low-level infection can reduce the quality of berries, which may affect the flavor of wine and table or raisin grape berries. Severe infection can cause berry cracking and dropping, or even result in significant loss of harvest (Gadoury et al. 2003; Qiu et al. 2015). Despite its importance, relatively little information is currently available regarding pathogenicity mechanisms of $E$. necator as well as other powdery mildew pathogens. Apart from the genetic intractability of all powdery mildew fungi, the lack of well-assembled genomes for the identification of key effectors of host-adapted powdery mildew species, subspecies, or isolates also hinders mechanistic studies of powdery mildew.

To date, the whole-genome sequences of five isolates of $E$. necator (Branching, C-strain, e1-101, Lodi, and Ranch9) have been reported. There are apparent sequence differences among the genomes of the five powdery mildew isolates with different genetic and geographical backgrounds (Jones et al. 2014). Another report indicated that there is sequence polymorphism between isolates from different regions and hosts in the eastern United States, let alone when the U.S. isolates are compared with those from southern France and Italy (Brewer and Milgroom 2010; Frenkel et al. 2012). Viticulture has a long history in China and there is rich wild grapevine germplasm (Gao et al. 2016) in many different regions of China. It is conceivable that the long-time grapevine-powdery mildew coevolution must have shaped the genomes of powdery mildew pathogens in these regions. Hence, obtaining the whole-genome sequence of a grapevine powdery mildew isolate identified in China would provide valuable sequence information for future investigation of host adaptation of powdery mildew in different regions.

Here, we report the genome sequence of an $E$. necator isolate NAFU1, which was isolated from Vitis vinifera 'Rizamat', Shaanxi Province, China and maintained on the susceptible grapevine cultivar Thompson Seedless (Gao et al. 2016). Spores E. necator NAFU1 were collected from infected leaves of grapevine at 10 to 15 days postinoculation using a small vacuum and used for genomic DNA extraction using the cetyltrimethylammonium bromide method (Feehan et al. 2017). Approximately $12 \mu \mathrm{g}$ of pure DNA with an average size of $20 \mathrm{~kb}$ was used for genome sequencing by the Oxford Nanopore and Illumina technologies. The genome sequences of $E$. necator NAFU1 were assembled by using a combination of short reads (approximately 6,657,136,974 bp) generated by Illumina sequencing (NOVASeq6000 platform, PE150, read length $=150 \mathrm{bp}$, paired-end reads) and long reads (approximately 13,268,751,137 bp) by Oxford Nanopore sequencing (PromethION) performed at Biomarker Technologies (Beijing, China). In total, $13.27 \mathrm{~Gb}$ of Nanopore long reads, representing approximately $217 \times$ coverage of the E. necator NAFU1 genome, and $6.66 \mathrm{~Gb}$ of Illumina NOVASeq6000 short reads (approximately 109x) were generated. The Nanopore reads of low quality and less than 2,000 bp were filtered out. NECAT was used to assemble the Nanopore subreads after filtering, and Pilon was used to correct the assembled sequences using the second-generation sequence data (Koren et al. 2017; Walker et al. 2014).

The final genome assembly of $E$. necator NAFU1 is $61.12 \mathrm{Mb}$ in length with $48.5 \% \mathrm{GC}$ content. The assembly contains 73 contigs with an $\mathrm{N}_{50}$ length of $2.06 \mathrm{Mb}$ (the longest contig length is $6.05 \mathrm{Mb}$ ) and $98.3 \%$ benchmarking universal single-copy ortholog (BUSCO) completeness (based on 1,315 conserved Ascomycota orthologs). This indicates that the wholegenome assembly of $E$. necator NAFU1 is of high quality, which ensures accurate prediction of the protein-coding genes in its genome. Genscan (Burge and Karlin 1997), Augustus v2.4 (Stanke and Waack 2003), GlimmerHMM v3.0.4 (Majoros et al. 2004), GenelD v1.4 (Blanco et al. 2007), and SNAP (version 2006-07-28) (Korf 2004) were used for ab initio gene prediction, and GeMoMa v1.3.1 (Keilwagen et al. 2016) was used for homology-based gene prediction. Finally, EVidenceModeler v1.1.1 (Haas et al. 2008) was used to integrate the above two methods to obtain 7,235 protein-coding genes. In total, 7,235 protein-coding genes were predicted, with an estimated BUSCO completeness of $96.7 \%$ and average length of proteincoding gene of $2,169 \mathrm{bp}$. The sequence features of the genome assembly of $E$. necator NAFU1 and other isolates are shown in Table 1. A comparison with the assembled genomes of other isolates (Jones et al. 2014) suggests a much deeper sequence depth and higher genome coverage, with fewer contigs, than for that of $E$. necator NAFU1.

To ensure a successful infection on their host plants, fungal pathogens produce a suite of carbohydrate-active enzymes (CAZymes) to digest polysaccharides of the plant cell wall and send hundreds of secreted effector proteins into the host cell to suppress plant immunity (Adachi et al. 2020; Yin et al. 2012). To assess the size of the CAZymes of E. necator 
Table 1. Sequence features of the genome assembly of Erysiphe necator NAFU1 and other isolates

\begin{tabular}{|c|c|c|c|c|c|c|}
\hline Genomic feature & NAFU1 & C-strain & Lodi & Ranch9 & Branching & e1-101 \\
\hline Sequencing platform & Nanopore & Illumina MiSeq & Illumina HiSeq & Illumina HiSeq & Illumina HiSeq & Illumina HiSeq \\
\hline BioSample & SAMN17620199 & SAMN02803834 & SAMN02803901 & SAMN02803894 & SAMN02803892 & SAMN02803896 \\
\hline BioProject & PRJNA695796 & PRJNA247407 & PRJNA248904 & PRJNA248903 & PRJNA248900 & PRJNA248902 \\
\hline Total assembly size (bp) & $61,122,667$ & $52,505,057$ & $49,793,988$ & $49,465,130$ & $50,658,153$ & $49,942,550$ \\
\hline Coverage & $217 x$ & $76 x$ & $42 x$ & $24 x$ & $29 x$ & $42 x$ \\
\hline GC content $(\%)$ & 48.5 & 39.0 & 38.9 & 38.8 & 38.5 & 38.8 \\
\hline Number of contigs & 73 & 8,584 & 8,093 & 8,274 & 11,631 & 7,601 \\
\hline Maximum contig length (bp) & $6,053,329$ & - & - & - & - & - \\
\hline Contig $\mathrm{N}_{50}(\mathrm{bp})$ & $2,063,233$ & 16,949 & 13,724 & 13,213 & 12,413 & 15,756 \\
\hline Contig $N_{90}(\mathrm{bp})$ & 822,440 & - & - & - & - & - \\
\hline Contig $L_{50}$ & 9 & 895 & 1,073 & 1,099 & 1,195 & 935 \\
\hline Total protein-coding genes & 7,235 & 6,484 & - & - & - & - \\
\hline BUSCO $(\%)^{a}$ & 98.3 & - & - & - & - & - \\
\hline Transfer RNAs & 244 & - & - & - & - & - \\
\hline Ribosomal RNAs & 76 & - & - & - & - & - \\
\hline Secretome ${ }^{b}$ & 453 & 422 & - & - & - & - \\
\hline Effectorome $^{c}$ & 196 & 150 & - & - & - & - \\
\hline
\end{tabular}

${ }^{a}$ BUSCO: benchmarking universal single-copy ortholog completeness.

${ }^{b}$ Secretome: proteins have signal peptide but without a transmembrane domain.

${ }^{\mathrm{c}}$ Effectorome: predicted by software EffectorP.

NAFU1, genes encoding such enzymes were predicted by the webtools at the CAZymes database. In total, 327 CAZyme genes were predicted in the genome of $E$. necator NAFU1. These CAZymes belong to five superfamilies, including 138 (42.2\%) glycosyl hydrolases, 96 (29.35\%) glycoside transferases, $53(16.2 \%)$ carbohydrate esterases, $26(7.95 \%)$ enzymes with auxiliary activities, and 14 (4.28\%) other carbohydrate-binding proteins To identify candidate genes encoding secreted proteins, SignalP v3.0 (Bendtsen et al. 2004) was used for the prediction of an N-terminal signal peptide and TMHMM (Krogh et al. 2001) was used for the prediction of transmembrane domains. In total, 453 genes in the genome were predicted to encode secreted proteins that contain a signal peptide but no transmembrane domains. Using EffectorP (Sperschneider et al. 2018), 41 genes were predicted to encode candidate effector proteins in the genome of $E$. necator NAFU1.

The availability of the genome sequence of E. necator NAFU1 will facilitate intraspecific as well as interspecific comparative genome analyses of powdery mildew fungi for investigating how they have coevolved with their respective plant hosts in different habitats. One future study we hope to conduct is to assess the impact of resistance from various wild Chinese grapevines on the effector repertoire of $E$. necator NAFU1 in comparison with $E$. necator originating in the United States and Europe.

\section{Data Availability}

The E. necator NAFU1 genome has been deposited at the DNA Data Bank of Japan/ European Nucleotide Archive/GenBank database under the accession number JAFBAW000000000 (BioProject PRJNA695796 and BioSample SAMN17620199). The version described in this article is version JAFBAW010000000.

\section{Author-Recommended Internet Resource}

CAZymes database: http://www.cazy.org

\section{Literature Cited}

Adachi, H., Biaas, A., and Kamoun, S. 2020. How to trick a plant pathogen? Biochemist (Lond.) 42:14-18.

Bendtsen, J. D., Nielsen, H., von Heijne, G., and Brunak, S. 2004. Improved prediction of signal peptides: SignalP 3.0. J. Mol. Biol. 340:783-795.

Blanco, E., Parra., G., and Guigó., R. 2007. Using geneid to identify genes. Curr. Protoc. Bioinf. 18:4.3.1-4.3.28.
Braun, U., and Cook, R. T. A. 2012. Taxonomic Manual of the Erysiphales (Powdery Mildews). American Phytopathological Society, St. Paul, MN, U.S.A.

Brewer, M. T., and Milgroom, M. G. 2010. Phylogeography and population structure of the grape powdery mildew fungus, Erysiphe necator, from diverse Vitis species. BMC Evol. Biol. 10:268.

Burge, C., and Karlin, S. 1997. Prediction of complete gene structures in human genomic DNA. J. Mol. Biol. 268:78-94. 
Dry, I. B., Feechan, A., Anderson, C., Jermakow, A. M., Bouquet, A., Adam-Blondon, A. F., and Thomas, M. R. 2010. Molecular strategies to enhance the genetic resistance of grapevines to powdery mildew. Aust. J. Grape Wine Res. 16: 94-105.

Feehan, J. M., Scheibel, K. E., Bourras, S., Underwood, W., Keller, B., and Somerville, S. C. 2017. Purification of high molecular weight genomic DNA from powdery mildew for long-read sequencing. J. Vis. Exp. 121:e55463.

Frenkel, O., Portillo, I., Brewer, M. T., Péros, J. P., Cadle-Davidson, L., and Milgroom, M. G. 2012. Development of microsatellite markers from the transcriptome of Erysiphe necator for analysing population structure in North America and Europe. Plant Pathol. 61:106-119.

Gadoury, D. M., Cadle-Davidson, L., Wilcox, W. F., Dry, I. B., Seem, R. C., and Milgroom, M. G. 2012. Grapevine powdery mildew (Erysiphe necator): A fascinating system for the study of the biology, ecology and epidemiology of an obligate biotroph. Mol. Plant Pathol. 13:1-16.

Gadoury, D. M., Seem, R. C., Ficke, A., and Wilcox, W. F. 2003. Ontogenic resistance to powdery mildew in grape berries. Phytopathology 93:547-555.

Gao, Y. R., Han, Y. T., Zhao, F. L., Li, Y. J., Cheng, Y., Ding, Q., Wang, Y. J., and Wen, Y. Q. 2016. Identification and utilization of a new Erysiphe necator isolate NAFU1 to quickly evaluate powdery mildew resistance in wild Chinese grapevine species using detached leaves. Plant Physiol. Biochem. 98:12-24.

Gessler, C., Pertot, I., and Perazzolli, M. 2011. Plasmopara viticola: A review of knowledge on downy mildew of grapevine and effective disease management. Phytopathol. Mediterr. 50:3-44.

Haas, B. J., Salzberg, S. L., Zhu, W., Pertea, M., Allen, J. E., Orvis, J., White, O., Buell, C. R., and Wortman, J. R. 2008. Automated eukaryotic gene structure annotation using EVidenceModeler and the Program to Assemble Spliced Alignments. Genome Biol. 9:R7.

Han, L., Weng, K., Ma, H., Xiang, G., Li, Z., Wang, Y., Liu, G., and Xu, Y. 2016. Identification and characterization of Erysiphe necator-responsive microRNAs in Chinese wild Vitis pseudoreticulata by high-throughput sequencing. Front. Plant Sci. 7:621.

Jones, L., Riaz, S., Morales-Cruz, A., Amrine, K. C., McGuire, B., Gubler, W. D., Walker, M. A., and Cantu, D. 2014. Adaptive genomic structural variation in the grape powdery mildew pathogen, Erysiphe necator. BMC Genomics 15: 1081.

Keilwagen, J., Wenk, M., Erickson, J. L., Schattat, M. H., Grau, J., and Hartung, F. 2016. Using intron position conservation for homology-based gene prediction. Nucleic Acids Res. 44:e89.

Koren, S., Walenz, B. P., Berlin, K., Miller, J. R., Bergman, N. H., and Phillippy, A. M. 2017. Canu: Scalable and accurate long-read assembly via adaptive k-mer weighting and repeat separation. Genome Res. 27:722-736.

Korf, I. 2004. Gene finding in novel genomes. BMC Bioinf. 5:59.

Krogh, A., Larsson, B., von Heijne, G., and Sonnhammer, E. L. 2001. Predicting transmembrane protein topology with a hidden Markov model: Application to complete genomes. J. Mol. Biol. 305:567-580.

Majoros, W. H., Pertea, M., and Salzberg, S. L. 2004. TigrScan and GlimmerHMM: Two open source ab initio eukaryotic gene-finders. Bioinformatics 20:2878-2879.

Qiu, W., Feechan, A., and Dry, I. 2015. Current understanding of grapevine defense mechanisms against the biotrophic fungus (Erysiphe necator), the causal agent of powdery mildew disease. Hortic. Res. 2:15020.

Sperschneider, J., Dodds, P. N., Gardiner, D. M., Singh, K. B., and Taylor, J. M. 2018. Improved prediction of fungal effector proteins from secretomes with EffectorP 2.0. Mol. Plant Pathol. 19:2094-2110.

Stanke, M., and Waack, S. 2003. Gene prediction with a hidden Markov model and a new intron submodel. Bioinformatics 19:ii215-ii225.

Walker, B. J., Abeel, T., Shea, T., Priest, M., Abouelliel, A., Sakthikumar, S., Cuomo, C. A., Zeng, Q., Wortman, J., Young, S. K., and Earl, A. M. 2014. Pilon: An integrated tool for comprehensive microbial variant detection and genome assembly improvement. PLoS One 9:e112963.

Wu, Y., Ma, X., Pan, Z., Kale, S. D., Song, Y., King, H., Zhang, Q., Presley, C., Deng, X., Wei, C. I., and Xiao, S. 2018. Comparative genome analyses reveal sequence features reflecting distinct modes of host-adaptation between dicot and monocot powdery mildew. BMC Genomics 19:705.

Yin, Y., Mao, X., Yang, J., Chen, X., Mao, F., and Xu, Y. 2012. dbCAN: A web resource for automated carbohydrate-active enzyme annotation. Nucleic Acids Res. 40:W445-W451. 